\title{
Estimation of incubation period distribution of COVID-19 using disease onset forward time: a novel cross-sectional and forward follow-up study
}

\author{
Jing Qin', Chong You ${ }^{2 *}$, Qiushi Lin ${ }^{2}$, Taojun Hü ${ }^{3}$, Shicheng Yü ${ }^{5}$ Xiao-Hua Zhou ${ }^{2,4,6 * *}$ \\ ${ }^{1}$ Biostatistics Research Branch, National Institute of Allergy and Infectious Diseases, National Institute of Health, Rockville, Maryland, United States, 20852. ${ }^{2 B e i j i n g}$ \\ International Center for Mathematical Research, Peking University, China, 100871. ${ }^{3}$ School of Mathematical Sciences, Peking University, China, 100871.4Department of \\ Biostatistics, School of Public Health, Peking University, China, $100871 .{ }^{5}$ Office for Epidemiology, Chinese Center for Disease Control and Prevention, China, $102206 .{ }^{6}$ Center \\ for Statistical Science, Peking University, China, 100871. \\ *Joint first author **Corresponding author. Email: azhou@math.pku.edu.cn
}

We propose a novel low-cost and accurate method to estimate the incubation distribution of COVID-19. We have conducted a cross-sectional and forward follow-up study by identifying those pre-symptomatic individuals at their time of departure from Wuhan and then following them until their symptoms developed. The renewal process is adopted by considering the incubation period as a renewal and the duration between departure and symptoms onset as a forward time. Such a method enhances the accuracy of estimation by reducing recall bias and utilizing the abundant and readily available data. The estimated median of incubation period is 7.76 days ( $95 \% \mathrm{Cl}: 7 \cdot 02-8 \cdot 53)$, the 90 th percentile is 14.28 days (95\% Cl: $13 \cdot 64-14 \cdot 90)$. By taking the possibility that a small portion of patients may contract the disease on their way out of Wuhan, the estimated probability that incubation period is longer than 14 days is between $5 \%$ to $10 \%$.

\section{Introduction}

The Center for Disease Control and Prevention (CDC) of China and World Health Organization (WHO) are closely monitoring the current outbreak of coronavirus disease 2019 (COVID-19). It was first identified in Wuhan, Hubei province, China, and has quickly spread across countries and become a global crisis. As of February 22, 2020, the National Health Commission (NHC) of China had confirmed a total of 76936 cases of COVID-19 in mainland China, including 2442 fatalities and 22888 recoveries (1). Various containment measures, including travel restrictions, isolation, and quarantine have been implemented in China with the aim to minimize virus transmission via human-to-human contact (2). Quarantine of individuals with exposure to infectious pathogens has always been an effective approach for containing contagious diseases in the past. One of the critical factors to determine the optimal quarantine of presymptomatic individuals is a good understanding of the incubation period, and this has been lacking for COVID-19.

The incubation period of an infectious disease is the time elapsed between infection and appearance of the first symptoms and signs. Precise knowledge of the incubation period would help to provide an optimal length of quarantine period for disease control purpose, and also is essential in the investigation of the mechanism of transmission and development of treatment. For example, the distribution of the incubation period is used to estimate the reproductive number $R$, that is, the average number of secondary infections produced by a primary case. The reproductive number is a key quantity that impacts the potential size of an epidemic. Despite the importance of the incubation period, it is often poorly estimated based on limited data.

To the best of our knowledge, there is only a handful of studies estimating the incubation period of COVID-19. Among them are Li et al. (3), Zhang et al. (4), Guan et al. (5), Backer et al. (6), Linton et al. (7) and Lauer et al. (8). The estimates of the incubation period from these five studies, together with other results of two other coronavirus disease, SARS and MERS, are listed in Table 1. In Li et al. (3), the first 425 lab-confirmed cases, reported as of January 22, 2020, were included in the study, while only 10 cases could be identified with the exact dates of exposure. The distribution of the incubation period was subsequently approximated by fitting a lognormal distribution to these ten data points, resulting in a mean incubation period of $5 \cdot 2$ days (95\% CI: $4 \cdot 1-7 \cdot 0)$, and the 95th percentile is 12.5 days. Similarly in Zhang et al. (4), 49 cases with no travel history who were identified by prospective contact tracing were used to estimate incubation period by fitting a lognormal distribution, resulting a mean incubation period of $5 \cdot 2$ days $(1 \cdot 8-12 \cdot 4)$. However, given the limited sample size, it is challenging to make a solid inference on the distribution of the incubation period. A different result was reported by Guan et al. (5), based on 291 patients who had clear information regarding the specific date of exposure as of January 29, 2020, stating that the median incubation period was 4.0 days (interquartile range, 2 to 7 ). However, 
such study of the incubation period can be highly influenced by the individuals' recall bias or interviewers' judgement on the possible dates of exposure rather than the actual dates of exposure that, in turn, might not be accurately monitored and determined, thus leading to a high percentage of error. In Backer et al. (6), 88 confirmed cases detected outside Wuhan were used to estimate the distribution of the incubation period. For each selected case, a censored interval for the incubation period can be obtained by travel history and symptoms onset. The distribution of the incubation period can then be estimated by fitting a Weibull, gamma, or lognormal distribution with censored data. However, this method contained two types of sampling biases: 1). with the longer incubation period, the patients who resided at Wuhan but developed symptoms outside Wuhan were easier to be observed (i.e., a patient with a shorter incubation period would develop symptoms before the planned trip and possibly cancel the trip, hence such case would not be observed) and therefore lead to an overestimation; 2). if the follow-up time (from infection to the end of the study) is short, only the shorter incubation period would be observed and hence lead to an underestimation (i.e., assume information of confirmed cases from Day 1 to Day 10 was collected, two patients, A and $\mathrm{B}$, both got infected on the day 5, Patient $\mathrm{A}$ had incubation period of 2 days while Patient $B$ had incubation period of 8 days, then only Patient A with the shorter incubation period would be included in the data, Patient $B$ with the longer incubation period would develop symptoms after Day 10 hence would not be included in the data). Linton et al. (7) proposed a similar approach to the study of Backer $e t a l$. with a larger sample size of 152, but in addition, corrected the second sampling bias aforementioned. However, the first problem in regard to sampling bias is still an unsolved issue. In Lauer et al. (8), a pooled data with sample size of 181 were used to estimate the incubation period. All collected cases in the pooled data had identifiable exposure and symptom onset windows available, of which 161 had a known recent history of travel to or residence in Wuhan which was the same kind of data collected in Backer et al. and Linton et al.; others had evidence of contact with travelers from Hubei or persons with known infection. A similar approach to Backer et al. was used, and the aforementioned two issues in regard to sampling bias remain unsolved. Lauer et al. reported that $2.5 \%$ patients developed symptoms after 11.5 days and claimed that it was highly unlikely that further symptomatic infections would be undetected after 14 days, while the same coauthors reported $5 \%$ patients have symptoms onset after 14 days in the study of Bi et al. (9).

To overcome the aforementioned problems, we propose a novel method to estimate the incubation period of COVID-19 by using the well-known renewal theory in probability (10). Such a method enhances the accuracy of estimation by reducing recall bias and utilizing abundance of the readily available forward time with a large sample size of 1084. To the best of our knowledge, this paper is a study of the distribution of the incubation period involving the largest number of samples to date. We find the estimated median of the incubation period is $7 \cdot 76$ days (95\% CI: $7 \cdot 02-8 \cdot 53)$, and mean is $8 \cdot 29$ days (95\% CI: $7 \cdot 67-8 \cdot 9$ ), the 90 th percentile is $14 \cdot 28$ days (95\% CI: $13 \cdot 64-14 \cdot 90$ ), and the 99th percentile is $20 \cdot 31$ days (95\% CI: 19·15-21.47). Furthermore, by taking the possibility that a small portion of patients may contract the disease on their way out of Wuhan, the estimated tail probability that incubation period is longer than 14 days is between $5 \%$ to $10 \%$. It is difficult to estimate the proportion of incubation beyond 14 days in general based on the small sample size. Compared with the existing published results, we are confident of our results since our sample size is much larger. Our estimated incubation period of COVID-19 is longer than the those given by previous researches on SARS, MERS, and COVID-19 in Table 1.

\section{Methods \\ Motivations}

As described in the previous section, the distribution of the incubation period in most of the literature is either described through a parametric model or its empirical distribution based on the observed incubation period from the contacttracing data. However, the contact-tracing data are challenging and expensive to obtain, and their accuracy can be highly influenced by recall bias. Hence, a low-cost and high-accuracy method to estimate the incubation distribution is needed. In this study, we make use of confirmed cases detected outside Wuhan with known histories of travel or residency in Wuhan to estimate the distribution of incubation. The renewal theory is implemented by treating an incubation period of a prevalence case as a renewal process. See more details of renewal process and corresponding assumptions in Supplement Material S1.

\section{Data collection and justification}

Publicly available data were retrieved from provincial and municipal health commissions in China and the ministries of health in other countries, including 12963 confirmed cases outside Hubei province as of February 15, 2020. Detailed information on confirmed cases includes region, gender, age, date of symptom onset, date of confirmation, history of travel or residency in Wuhan, and date of departure from Wuhan. The date of symptoms onset in these data refers to the date reported by the patient on which the clinical symptoms first appeared, where the clinical symptoms include fever, cough, nausea, vomiting, diarrhea, and others. Among 12963 confirmed cases, 6345 cases had their dates of symptom onset collected; 3169 cases had histories of travel or residency in 
Wuhan; 2514 cases had their dates of departure recorded; and 1922 cases had records of both dates of departure from Wuhan and dates of symptoms onset. However, not all 1922 cases should be taken in the analysis. After examining the collected data, there were a total of 1084 cases that meet the criteria described in Supplement S2 and were followed forwardly.

Figure 1 shows the design of the cross-sectional and forward follow-up study. The dot on the left end of each segment is a date of infection, while the square on the right end is a date of symptoms onset. The date of departure from Wuhan cuts the line segment in between. Note that only solid lines were followed in our cohort, while dashed lines are not in the cohort because the date of departure from Wuhan is not between January 19, 2020, and January 23, 2020.

Among the 1084 cases with gender information in the study, $468(43.30 \%)$ are female. The mean age of patients was 41.31 and the median age was 40 . Over $80 \%$ of the cases were between 20 and 60 . The youngest confirmed case in our cohort was six months-old while the oldest was 86 years-old. Table 2 shows the demographic characteristics of patients with COVID-19 in the Wuhan departure cohort and the entire data collected as of February 15, 2020. Though there are slight differences between the selected cases and all case, we explored the correlation between forward time and age instead and found the correlated between forward time and age was -0.0309 . Hence there is no evidence that the incubation time depends on age in this dataset, and the observed forward times should be able to represent the that of in the general population. More demographic characteristics of patients are summarized in Supplement S2.

\section{Estimation of incubation period distribution of COVID-19}

Let $Y$ be the incubation period of an infected case with probability density function $f(y)$ where $y>0$. Let $A$ be the duration from infection in Wuhan to the departure of Wuhan, which can be considered as the backward time in a renewal process. Let $V$ denote the duration between departure from Wuhan and onset of symptoms, which can be considered as the forward time in a renewal process. Then $V$ has the density as follows,

$$
g(v)=\frac{\bar{F}(v)}{\mu}, v \geq 0
$$

where $\bar{F}(\cdot)$ is the survival function corresponding to $f(\bullet)$ and $\mu=\int_{0}^{\infty} y f(y) d y$ is the mean incubation period. Note that $A$ and $V$ have the same density marginally and the aforementioned sampling bias can be corrected by using Eq. (1). See more technical details in Supplement S3.
In our cohort of COVID-19 cases, we assume the incubation period is a Weibull random variable, the estimates in Weibull model can be obtained by maximizing the corresponding likelihood function. The mean and percentiles of the incubation period can be calculated from the parametric Weibull distribution. The confidence intervals in this study are obtained using bootstrap method with $\mathrm{B}=1000$ resamples. Note that Gamma distribution and log-normal distribution are also fitted for the incubation, both provide similar estimates of quantiles compared with Weibull.

\section{Sensitivity analysis}

It is arguable that people who left Wuhan might also be infected on the day of departure since they had a higher chance to be exposed to this highly contagious, human-to-humantransmitted virus in a crowded environment as cases were increasing. In such case, the duration between departure from Wuhan and onset of symptoms is no longer only the forward time, but a mixture of the incubation period and the forward time. Unfortunately, it is unclear who got infected before departure and who got infected at the event of departure. Hence, a mixture sensitivity forward time model is proposed, that is,

$$
h(v)=\alpha \lambda\left\{\pi(v \lambda)^{\alpha-1}+(1-\pi) / \Gamma(1 / \alpha)\right\} \exp \left(-(v \lambda)^{\alpha}\right), v \geq 0
$$

If $\alpha \neq 1$, it is possible to identify all underlying parameters. We explore the sensitivity of estimates of incubation period by assuming a range of $\pi$, that is $\pi=0,0.05,0.1$, and 0.2 , and estimate $\alpha$ and $\lambda$ by maximizing the product of likelihoods, $\prod_{i=1}^{I} h\left(v_{i}\right)$, with respect to $\alpha$ and $\lambda$.

\section{Results}

By fitting the observed forward times $v_{i}$ of the 1084 cases in our cohort to the likelihood function (4), we find that $\pi=0$ gives the largest loglikelihood, hence we set $\pi=0$ as the reference scenario. The maximum likelihood estimates are $\hat{\alpha}=1.97(95 \%$ CI: $1 \cdot 75-2 \cdot 28)$ and $\hat{\lambda}=0.11$ (95\% CI: $0 \cdot 10-0 \cdot 12)$ in our reference scenario. The estimated 5th, 25th, 50th, 75th, 90th, 95th, 99th, and 99.9th percentiles of the incubation period are $2 \cdot 07$ (95\% CI: $1 \cdot 60-2 \cdot 69$ ), 4.97 (95\% CI: $4 \cdot 25-5 \cdot 78$ ), $7 \cdot 76$ (95\% CI: $7 \cdot 02-8 \cdot 53)$, $11 \cdot 04$ (95\% CI: 10·34-11.66), $14 \cdot 28$ (95\% CI: 13.64-14.90), 16.32 (95\% CI: 15.62-17.04), $20 \cdot 31$ (95\% CI: $19 \cdot 15-21 \cdot 47)$, and $24 \cdot 95$ (95\% CI: $23 \cdot 04-26 \cdot 81)$ days, respectively. The mean incubation period is $8 \cdot 29$ (95\% CI: $7 \cdot 67-8 \cdot 90)$ days. Estimates based on Gamma distribution and log-normal distribution provide very similar results, where the 50th percentile is 8.16 and 8.42 respectively, the 90th percentile is 14.23 and respectively 14.11 , and the log likelihoods are 2843.34 and -2845.57 which are slightly smaller comparing with Weibull distribution. The average time from leaving Wuhan to symptom onset is $5 \cdot 30$ days, the sample median is 5 
days, and the maximum is 22 days. Figure 2 visualizes the fitted density function in Eq. (2) in a solid line onto the histogram of observed forward times, and the dashed line is the Weibull probability density function in Eq. (1) for incubation period distribution. Note that Eq. (2) fits the observed forward times well, suggesting that our model is reasonable and the results are therefore trustworthy.

Table 3 summaries the estimates of parameters, and the mean and percentiles of incubation period. We can see that the estimates for mean and percentiles decrease as the proportion of people who got infected at the event of departure, $\pi$, increases. However, variation of the results from $\pi=0$ to 0.2 is only about one day which we believe is still in an acceptable range.

\section{Discussion}

A sound estimate of the distribution of the incubation period plays a vital role in epidemiology. Its application includes decisions regarding the length of quarantine for prevention and control, dynamic models that accurately predict the disease process, and determining the contaminated source in foodborne outbreaks. In this paper, we propose a novel method to estimate the incubation distribution which only requires information on travel histories and dates of symptoms onset. This method enhances the accuracy of estimation by reducing recall bias and utilizing abundance of the readily available forward time data. To the best of our knowledge this study of incubation period involves the largest number of samples to date. In addition, this is the first article to consider the incubation period for COVID-19 as a renewal process which is a well-studied methodology and has a solid theoretical foundation. The estimated incubation period has a median of $7 \cdot 76$ days (95\% CI: $7 \cdot 02-8 \cdot 53$ ), a mean of 8.29 days (95\% CI: $7 \cdot 67-8 \cdot 90)$, the 90 th percentile is $14 \cdot 28$ days $(95 \%$ CI: $13 \cdot 64-$ $14.90)$, and the 99 th percentile is 20.31 days (95\% CI: $19 \cdot 15-$ $21.47)$. By taking the possibility that a small portion of patients may contract the disease on their way out of Wuhan, the estimated tail probability that incubation period is longer than 14 days is between $5 \%$ to $10 \%$. Compared with the results published in Li et al., Guan et al., Backer et al., and Linton et $a l$., the incubation period estimated in our study is significantly longer (3-7). Below is some evidence that may potentially support our findings of the long incubation period:

1. In the study of Guan et al. (4) on behalf of China Medical Treatment Expert Group for COVID-19, the incubation period had a reported median of 4 days, the first quartile of 2 days and the third quartile of 7 days. By fitting a commonly used Weibull distribution to such quartiles, we can obtain $\hat{\alpha}=$ 1.24 and $\hat{\lambda}=0.186$ defined in Eq. (2). As a consequence, the estimated $90 \%, 95 \%$ and $99 \%$ percentiles are, respectively, $10.54,13.04$ and 18.45 days, which indicates that some patients may have extended incubation periods. In addition, in the commentary published in NEJMqianyan by the authors of Guan et al., it was reported that the incubation period of one patient in each of the severe and non-severe groups was up to 24 days, 13 cases $(12 \cdot 7 \%)$ with an incubation period greater than 14 days and 8 cases $(7 \cdot 3 \%)$ with an incubation period greater than 18 days which were close to what have found in our study (11).

2. One particular case reported by Yibin municipal health commissions in China stated that a 64-year-old female was diagnosed with COVID-19 on February 11, 2020 at Yibin, Sichuan province 20 days after returning from Wuhan. This patient was under self-quarantine at home with the family for 18 days, from January 23 to February 9. On February 8, the patient developed mild symptoms of cough with sputum production (12).

3. It was reported in Bai et al. (13), the incubation period for patient 1 was 19 days. However, the claimed 19-day incubation was the time difference between departure from $\mathrm{Wu}$ han and symptoms onset, namely the forward time in our study. The actual incubation period should be longer than 19 days.

Based on the estimated incubation distribution in this study, about $10 \%$ of patients with COVID-19 would develop symptoms after 14 days of infection. This may be a public health concern in regard to the current 14-day quarantine period. Our approach does require that certain assumptions to be met, which we detail below.

1. The collection of forward time depends on the followup time, that is, if the follow-up time is not long enough, we would only be able to include those with a shorter incubation period in the Wuhan departure cohort. This may lead to an underestimation of the incubation period. The same limitation also applies to Backer et al. and Linton et al. $(6,7)$. However, as explained earlier, we only included cases who left Wuhan before January 23 in this study, which leaves an average follow-up time of 25 days. Hence it is less likely we missed those patients with longer incubation periods based on the largest incubation period of 24 days reported in Guan et al. (5). Note that the 24-day incubation period was reported as an outlier in Guan et al.

2. We assume that the individuals included in our cohort were either infected in Wuhan or on the way to their destination from Wuhan, violation of such assumption lead to an overestimation of incubation period. The same limitation also applies to Backer et al., Linton et al. and Lauer et al. (6, 7). However, with a carefully selected cohort justified in the section of Method, the chance for an individual in the Wuhan departure cohort getting infected outside Wuhan should be relatively small. Nonetheless, we acknowledge this possibility exists, for example, a family member could be uninfected by the time of departing Wuhan but got infected by other family members or outside contacts after leaving Wuhan. A 
sensitivity analysis was also conducted by removing all cases who left Wuhan with their families in the Wuhan departure cohort, and we found it only resulted in a small change of the estimated distribution of the incubation period.

3. Individuals in our selected cohort were those who got infected in the early days of the outbreak. They were likely the first- or second-generation cases. Our results do not apply to higher generation cases if the virus mutates.

\section{REFERENCES AND NOTES}

1. National Health Commission of the People's Republic of China, National Health Commission update on February 23, 2020 [Internet]. Beijing, CN: National Health Commission of the People's Republic of China; 2020 Feb [cited 2020 Feb 27]. Available http://weekly.chinacdc.cn/news/TrackingtheEpidemic.htm\#NHCFeb23.

2. Chinese Center for Disease Control and Prevention, Epidemic update and risk assessment of 2019 Novel Coronavirus [Internet]. Beijing (CN): Chinese Center for Disease Control and Prevention; 2020 Jan 28 [cited 2020 Feb 27]. Available from:

http://www.chinacdc.cn/jkzt/crb/zl/szkb_11803/jszl_11811/202001/P020200 128523354919292.pdf.

3. Q. Li, X. Guan, P. Wu, X. Wang, L. Zhou, Y. Tong, R. Ren, K. S. M. Leung, E. H. Y. Lau, J. Y. Wong, X. Xing, N. Xiang, Y. Wu, C. Li, Q. Chen, D. Li, T. Liu, J. Zhao, M. Liu, W. Tu, C. Chen, L. Jin, R. Yang, Q. Wang, S. Zhou, R. Wang, H. Liu, Y. Luo, Y. Liu, G. Shao, H. Li, Z. Tao, Y. Yang, Z. Deng, B. Liu, Z. Ma, Y. Zhang, G. Shi, T. T. Y. Lam, J. T. Wu, G. F. Gao, B. J. Cowling, B. Yang, G. M. Leung, Z. Feng, Early Transmission Dynamics in Wuhan, China, of Novel Coronavirus-Infected Pneumonia. N. Engl. J. Med. 382, 1199-1207 (2020). doi:10.1056/NEJMoa2001316 Medline

4. J. Zhang, M. Litvinova, W. Wang, Y. Wang, X. Deng, X. Chen, M. Li, W. Zheng, L. Yi, X. Chen, Q. Wu, Y. Liang, X. Wang, J. Yang, K. Sun, I. M. Longini Jr., M. E. Halloran, P. Wu, B. J. Cowling, S. Merler, C. Viboud, A. Vespignani, M. Ajelli, H. Yu, Evolving epidemiology and transmission dynamics of coronavirus disease 2019 outside Hubei province, China: A descriptive and modelling study. Lancet Infect. Dis. 20, 793-802 (2020). doi:10.1016/S1473-3099(20)30230-9 Medline

5. W. J. Guan, Z. Y. Ni, Y. Hu, W. H. Liang, C. Q. Ou, J. X. He, L. Liu, H. Shan, C. L. Lei, D. S. C. Hui, B. Du, L. J. Li, G. Zeng, K.-Y. Yuen, R. C. Chen, C. L. Tang, T. Wang, P. Y. Chen, J. Xiang, S. Y. Li, J. L. Wang, Z. J. Liang, Y. X. Peng, L. Wei, Y. Liu, Y. H. Hu, P. Peng, J. M. Wang, J. Y. Liu, Z. Chen, G. Li, Z. J. Zheng, S. Q. Qiu, J. Luo, C. J. Ye, S. Y. Zhu, N. S. Zhong; China Medical Treatment Expert Group for Covid-19, Clinical Characteristics of Coronavirus Disease 2019 in China. N. Engl. J. Med. 382, 17081720 (2020). doi:10.1056/NEJMoa2002032 Medline

6. J. A. Backer, D. Klinkenberg, J. Wallinga, Incubation period of 2019 novel coronavirus (2019-nCoV) infections among travellers from Wuhan, China, 20-28 January 2020. Euro Surveill. 25, 2000062 (2020). doi:10.2807/15607917.ES.2020.25.5.2000062 Medline

7. N. M. Linton, T. Kobayashi, Y. Yang, K. Hayashi, A. R. Akhmetzhanov, S. M. Jung, B. Yuan, R. Kinoshita, H. Nishiura, Incubation Period and Other Epidemiological Characteristics of 2019 Novel Coronavirus Infections with Right Truncation: A Statistical Analysis of Publicly Available Case Data. J. Clin. Med. 9, 538 (2020). doi:10.3390/jcm9020538 Medline

8. S. A. Lauer, K. H. Grantz, Q. Bi, F. K. Jones, Q. Zheng, H. R. Meredith, A. S. Azman, N. G. Reich, J. Lessler, The Incubation Period of Coronavirus Disease 2019 (COVID-19) From Publicly Reported Confirmed Cases: Estimation and Application. Ann. Intern. Med. 172, 577-582 (2020). doi:10.7326/M20-0504 $\underline{\text { Medline }}$

9. Q. Bi et al., Epidemiology and Transmission of COVID-19 in Shenzhen China: Analysis of 391 cases and 1,286 of their close contacts. Lancet Infect. Dis. 20, e148 (2020).

10. W. Feller, An introduction to probability theory and its applications (3rd edition). New York: Wiley; 1968.

11. W.-j. Guan, W.-h. Liang, J.-h. He, N.-S. Zhong, Clarifying of Clinical Characteristics of Coronavirus Disease 2019 in China. Available from:
https://nejmqianyan.cn/article/YXQYoa2002032?sg=AbW1NGsHw3NxPd6F

12. Daily updates of COVID-19 in Yibin. YBW JW [Internet]. 2020 Feb 11 [cited 2020 Feb 27]. Available from: http://ybwjw.yibin.gov.cn/gzdt/202002/t20200212 1220372.html.

13. Y. Bai, L. Yao, T. Wei, F. Tian, D.-Y. Jin, L. Chen, M. Wang, Presumed Asymptomatic Carrier Transmission of COVID-19. JAMA 323, 1406-1407 (2020). doi:10.1001/jama.2020.2565 Medline

14. E. H. Lau, C. A. Hsiung, B. J. Cowling, C.-H. Chen, L.-M. Ho, T. Tsang, C.-W. Chang, C. A. Donnelly, G. M. Leung, A comparative epidemiologic analysis of SARS in Hong Kong, Beijing and Taiwan. BMC Infect. Dis. 10, 50 (2010). doi:10.1186/1471-233410-50 Medline

15. V. Virlogeux, V. J. Fang, M. Park, J. T. Wu, B. J. Cowling, Comparison of incubation period distribution of human infections with MERS-CoV in South Korea and Saudi Arabia. Sci. Rep. 6, 35839 (2016). doi:10.1038/srep35839 Medline

16. J.-E. Park, S. Jung, A. Kim, J.-E. Park, MERS transmission and risk factors: A systematic review. BMC Public Health 18, 574 (2018). doi:10.1186/s12889-0185484-8 Medline

17. G. M. Leung, L. M. Ho, T. H. Lam, A. J. Hedley, Epidemiology of SARS in the 2003 Hong Kong epidemic. Hong Kong Med. J. 15 (Suppl 9), 12-16 (2009). Medline

18. Q.-C. Cai, Q.-F. Xu, J.-M. Xu, Q. Guo, X. Cheng, G.-M. Zhao, Q.-W. Sun, J. Lu, Q.-W. Jiang, Refined estimate of the incubation period of severe acute respiratory syndrome and related influencing factors. Am. J. Epidemiol. 163, 211-216 (2006). doi:10.1093/aje/kwi034 Medline

19. B. J. Cowling, M. Park, V. J. Fang, P. Wu, G. M. Leung, J. T. Wu, Preliminary epidemiological assessment of MERS-CoV outbreak in South Korea, May to June 2015. Euro Surveill. 20, 7-13 (2015). Medline

20. A. Y. Kuk, S. Ma, The estimation of SARS incubation distribution from serial interval data using a convolution likelihood. Stat. Med. 24, 2525-2537 (2005). doi:10.1002/sim.2123 Medline

21. C. A. Donnelly, A. C. Ghani, G. M. Leung, A. J. Hedley, C. Fraser, S. Riley, L. J. AbuRaddad, L.-M. Ho, T.-Q. Thach, P. Chau, K.-P. Chan, T.-H. Lam, L.-Y. Tse, T. Tsang, S.-H. Liu, J. H. B. Kong, E. M. C. Lau, N. M. Ferguson, R. M. Anderson, Epidemiological determinants of spread of causal agent of severe acute respiratory syndrome in Hong Kong. Lancet 361, 1761-1766 (2003). doi:10.1016/S0140-6736(03)13410-1 Medline

22. M. I. Meltzer, Multiple contact dates and SARS incubation periods. Emerg. Infect. Dis. 10, 207-209 (2004). doi:10.3201/eid1002.030426 Medline

23. A. Assiri, A. McGeer, T. M. Perl, C. S. Price, A. A. Al Rabeeah, D. A. T. Cummings, Z. N. Alabdullatif, M. Assad, A. Almulhim, H. Makhdoom, H. Madani, R. Alhakeem, J. A. Al-Tawfiq, M. Cotten, S. J. Watson, P. Kellam, A. I. Zumla, Z. A. Memish; KSA MERS-CoV Investigation Team, Hospital outbreak of Middle East respiratory syndrome coronavirus. N. Engl. J. Med. 369, 407-416 (2013). doi:10.1056/NEJMoa1306742 Medline

24. H. Y. Park, E. J. Lee, Y. W. Ryu, Y. Kim, H. Kim, H. Lee, S. J. Yi, Epidemiological investigation of MERS-CoV spread in a single hospital in South Korea, May to June 2015. Euro Surveill. 20, 1-6 (2015). doi:10.2807/1560-7917.ES2015.20.25.21169 Medline

25. J. Qin, Biased Sampling, Over-identified Parameter Problems and Beyond. Singapore: Springer Singapore. (2017).

26. Q Lin, T Hu, X-HZhou, Estimating the daily trends in the size of 2019-nCoV infected population in Wuhan. Infect. Dis. Poverty 9, 69 (2020) doi: https://doi.org/10.1101/2020.02.12.20022277.

27. China declares lockdown in Wuhan on Thursday due to coronavirus outbreak. TASS [Internet]. 2020 Jan 22 [cited 2020 Feb 27]. Available from: https://tass.com/world/1111981.

\section{ACKNOWLEDGMENTS}

We thank Dr. Dean Follmann from National Institute of Allergy and Infectious Diseases for comments that greatly improved the manuscript, Dr. Weigong Zhou from U.S. Centers for Disease Control and Prevention (CDC) and Dr. Mary Thompson from University of Waterloo for many helpful comments and suggestions. We also thank Benjamin Snow, ELS, from Leidos Biomedical Research, Inc for providing a technical review of the manuscript. Funding: This research is supported by National Natural Science Foundation of China grant 8204100362 and Zhejiang University special scientific research fund for COVID- 
19 prevention and control. Competing Interests: The authors declare that they have no competing interests. Author Contributions: J.Q.: Study design, writing, and data interpretation. C.Y.: Writing, literature search, data interpretation. Q. L.:

Writing, data analysis, and data collection. T. H.: Data analysis and data

collection. S. Y.: Data interpretation. X.-H. Z.: Study design, writing, and data

interpretation. Data and materials availability: Data and codes are now

available from https://github.com/johnnyhu149/estimating incubation period

\section{SUPPLEMENTARY MATERIALS}

advances. sciencemag.org/cgi/content/full/sciadv.abc1202/DC1

Submitted 8 April 2020

Accepted 2 July 2020

Published First Release 7 August 2020

10.1126/sciadv.abc1202 


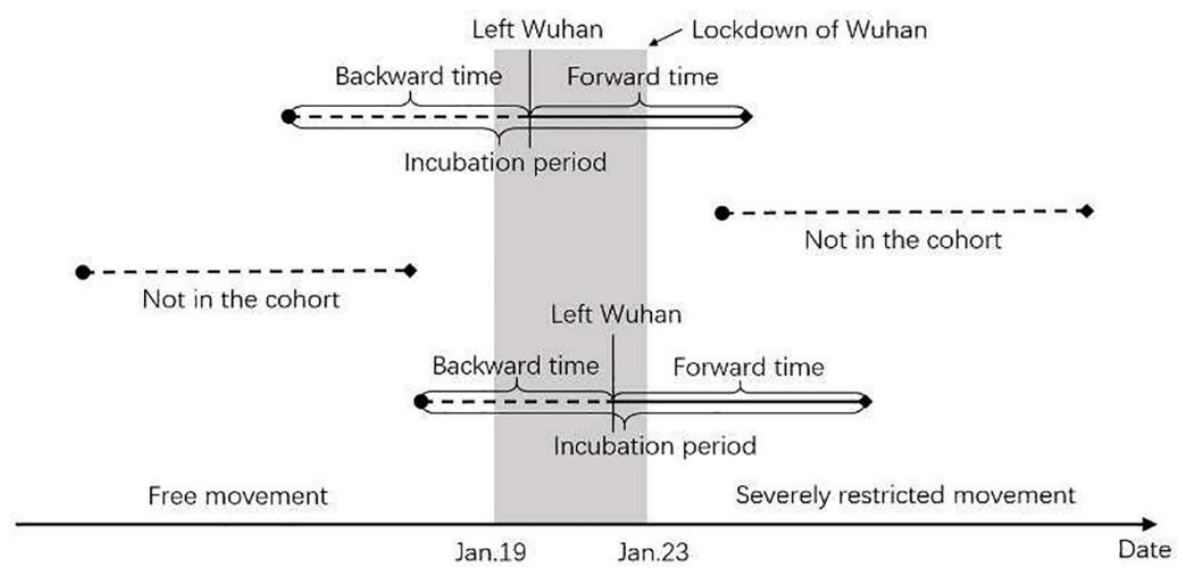

Fig. 1. Illustration of our cross-sectional and forward follow-up study. Backward and incubation periods are not observed, while Wuhan departure and forward time are observed. 


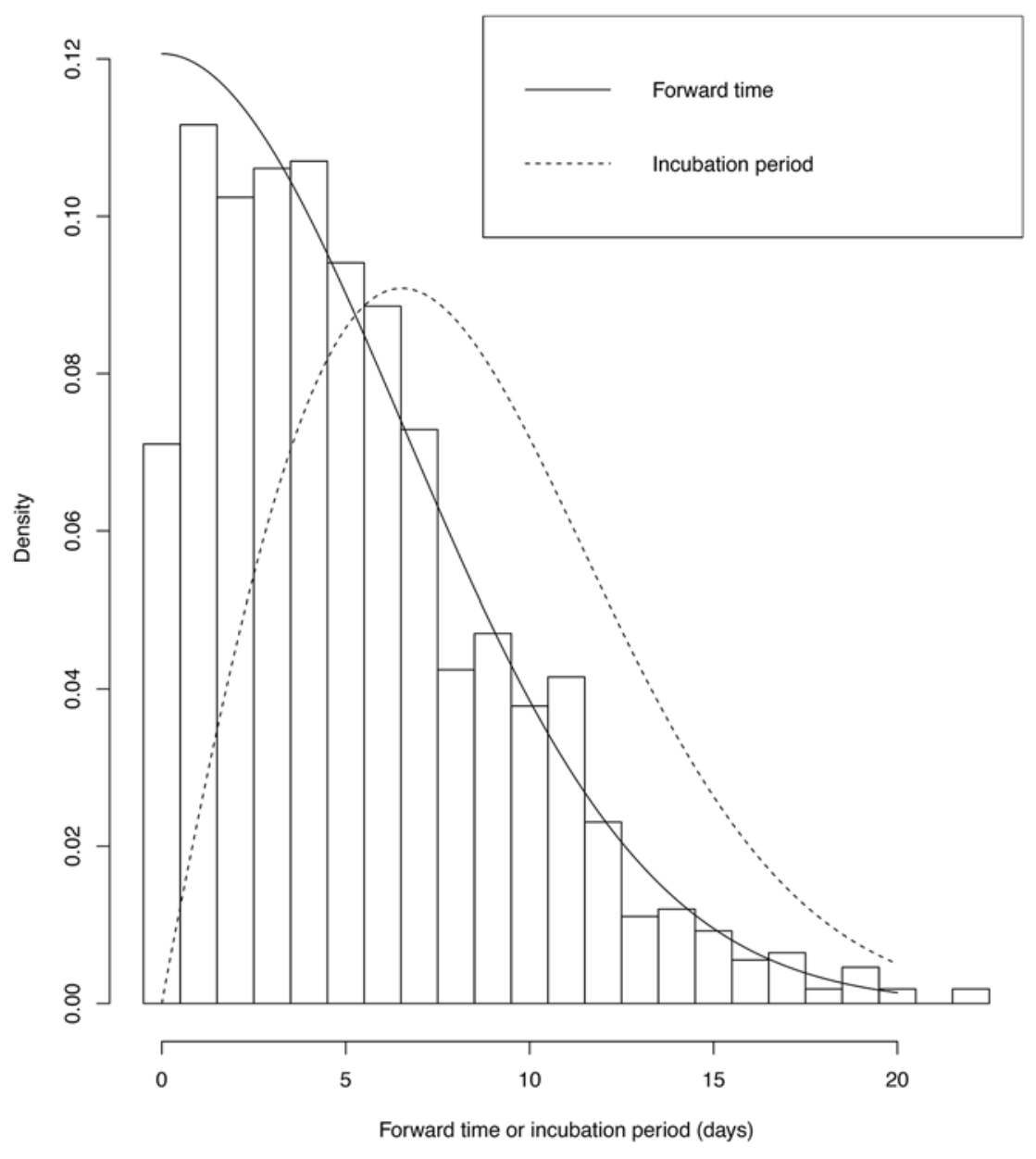

Fig. 2. Histogram and estimated probability density functions for the time from Wuhan departure to symptoms onset, i.e., forward time. 
Table 1. Estimates for the incubation periods of SARS, MERS, and COVID-19.

\begin{tabular}{|c|c|c|c|}
\hline $\begin{array}{l}\text { Incubation } \\
\text { distribution } \\
\text { metric }\end{array}$ & SARS & MERS & COVID-19 \\
\hline $\begin{array}{l}\text { Mean (SD) or } \\
\text { Mean (95\% } \\
\mathrm{Cl})\end{array}$ & $\begin{array}{l}\text { Hong Kong (14): } 4 \cdot 4(4 \cdot 6) ; \\
\text { Beijing (14): 5.7 (9.7); } \\
\text { Taiwan (14): } 6 \cdot 9(6 \cdot 1) \\
\text { Hong Kong (17): } 4 \cdot 6(15 \cdot 9) \\
\text { Mainland China (20): 5.29 (12.33); } \\
\text { Singapore (21): } 4 \cdot 83(4 \cdot 37-5 \cdot 29) ; \\
\text { Hong Kong (21): } 6 \cdot 37(5 \cdot 29-7 \cdot 75)\end{array}$ & $\begin{array}{l}\text { Saudi Arabia (15): } 5.0 \text { (4.0- } \\
6 \cdot 6) ; \\
\text { South Korea (15): } 6.9 \text { (6.3- } \\
7 \cdot 5) ; \\
\text { South Korea (16): } 6.9 \text { (6.3- } \\
7 \cdot 5) ; \\
\text { Saudi Arabia (16): } 5.0 \text { (4.4- } \\
6 \cdot 6) ; \\
\text { South Korea (19): } 6 \cdot 7 \text { (6.1- } \\
7 \cdot 3)\end{array}$ & 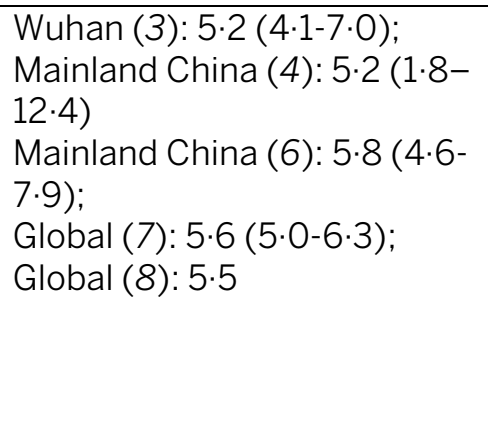 \\
\hline $\begin{array}{l}\text { Median or } \\
\text { Median (95\% } \\
\text { Cl) }\end{array}$ & Hong Kong, Canada, USA (22): 4 & $\begin{array}{l}\text { South Korea (19): } 6 \cdot 0 \text { (4.0- } \\
7 \cdot 0) \text {; } \\
\text { Middle East (23): } 5 \cdot 2 \text { (1.9- } \\
\text { 14.7); } \\
\text { South Korea (24): } 6 \cdot 3 \text { (5.7- } \\
6 \cdot 8)\end{array}$ & $\begin{array}{l}\text { Mainland China (5): 3.0; } \\
\text { Global (8): 5.1 (4.5-5.8) }\end{array}$ \\
\hline Percentiles & $\begin{array}{l}\text { Mainland China, 90\% (18): 10.7; } \\
\text { Hong Kong, Canada, USA, 90\% } \\
\text { (22): 12; } \\
\text { Singapore, 95\% (20): } 9.66(0 \cdot 5) ; \\
\text { Mainland China, 95\% (18): 13.91; } \\
\text { Hong Kong, 95\% (21): 14.22; } \\
\text { Mainland China, 99\% (18): } 20 \cdot 08\end{array}$ & NA & $\begin{array}{l}\text { Mainland China, 2.5\% (6): 1.3; } \\
\text { Wuhan, 95\% (3): 12.5; } \\
\text { Mainland China, } 97 \cdot 5 \%(6) \text { : } \\
11 \cdot 3 \\
\text { Global, } 2 \cdot 5 \%(8): 2 \cdot 2(1.8-2 \cdot 9) \\
\text { Global, } 97 \cdot 5 \%(8): 11 \cdot 5(8 \cdot 2- \\
15 \cdot 6)\end{array}$ \\
\hline
\end{tabular}

COVID-19=coronavirus disease 2019. MERS=Middle East respiratory syndrome. SARS=severe acute respiratory syndrome. 
Table 2. Comparison between the demographic characteristics of patients with COVID-19 in the studying cohort and all cases collected as of February 15, 2020.

\begin{tabular}{lllllll}
\hline $\begin{array}{l}\text { Age group } \\
\text { (years) }\end{array}$ & $\begin{array}{l}\text { Female } \\
\text { Studying co- } \\
\text { hort }\end{array}$ & All cases & $\begin{array}{l}\text { Male } \\
\text { Studying } \\
\text { cohort }\end{array}$ & All cases & $\begin{array}{l}\text { No information } \\
\text { Studying } \\
\text { cohort }\end{array}$ & All cases \\
& $468(43 \cdot 3)^{*}$ & $\begin{array}{l}4121 \\
(47 \cdot 3)\end{array}$ & $\begin{array}{l}614(57 \cdot 1) \\
126(3 \cdot 2)\end{array}$ & $\begin{array}{l}4597 \\
(52 \cdot 7)\end{array}$ & 2 & 4245 \\
$0-19$ & $17(3 \cdot 7)$ & $180(4 \cdot 2)$ & 0 & 3 \\
\hline $20-39$ & $189(40 \cdot 9)$ & $\begin{array}{l}1250 \\
(32 \cdot 2)\end{array}$ & $292(48 \cdot 3)$ & 1508 & 1 & 48 \\
$40-59$ & $195(42 \cdot 2)$ & $\begin{array}{l}1667 \\
(43 \cdot 0)\end{array}$ & $226(37 \cdot 4)$ & $\begin{array}{l}(35 \cdot 0) \\
(843\end{array}$ & 0 & 57 \\
$60-79$ & $60(13 \cdot 0)$ & $\begin{array}{l}749 \\
(19 \cdot 3)\end{array}$ & $62(10 \cdot 2)$ & $701(16 \cdot 3)$ & 0 & 40 \\
$\geq 80$ & $1(0 \cdot 2)$ & $85(2 \cdot 2)$ & $1(0 \cdot 2)$ & $78(1 \cdot 8)$ & 0 & 8 \\
No information & 6 & 244 & 9 & 287 & 0 & 4089
\end{tabular}

* Number (\%). The percentages do not take missing data into account.

Table 3. Results of our model based on different choices of $\pi$.

\begin{tabular}{|c|c|c|c|c|}
\hline \multirow[t]{2}{*}{ Scenario } & \multirow{2}{*}{$\begin{array}{l}\text { Reference case } \\
\pi=0\end{array}$} & \multicolumn{3}{|c|}{ Additional \% infected on the Wuhan departure day } \\
\hline & & $\pi=5 \%$ & $\pi=10 \%$ & $\pi=20 \%$ \\
\hline$\hat{\alpha}$ & $1.97(1.75,2.28)$ & $1.93(1.72,2.22)$ & $1.89(1.69,2.12)$ & $1.81(1.66,2.02)$ \\
\hline$\hat{\lambda}$ & $0.11(0.1,0.12)$ & $0.11(0.1,0.12)$ & $0.11(0.11,0.12)$ & $0.12(0.11,0.13)$ \\
\hline Mean & $8.29(7.67,8.9)$ & $8.01(7.45,8.61)$ & $7.75(7.23,8.31)$ & $7.32(6.85,7.8)$ \\
\hline $5 \%$ & $2.07(1.6,2.69)$ & $1.93(1.5,2.52)$ & $1.81(1.42,2.3)$ & $1.60(1.29,2)$ \\
\hline $25 \%$ & $4.97(4.25,5.78)$ & $4.73(4.07,5.49)$ & $4.51(3.92,5.19)$ & $4.14(3.66,4.7)$ \\
\hline Median & $7.76(7.02,8.53)$ & $7.47(6.78,8.18)$ & $7.19(6.55,7.9)$ & $6.73(6.19,7.3)$ \\
\hline $75 \%$ & $11.04(10.34,11.66)$ & $10.7(10.07,11.35)$ & $10.38(9.78,10.98)$ & $\begin{array}{l}9.86(9.3,10.4) \\
13.04(12.44\end{array}$ \\
\hline $90 \%$ & $14.28(13.64,14.9)$ & $13.92(13.32,14.57)$ & $13.59(12.99,14.17)$ & 13.59) \\
\hline $95 \%$ & $16.32(15.62,17.04)$ & $\begin{array}{l}\text { 15.95(15.3, 16.65) } \\
\text { 19.94(18.87, }\end{array}$ & $15.62(14.91,16.26)$ & $\begin{array}{l}\text { 15.07(14.38, } \\
15.72)\end{array}$ \\
\hline $99 \%$ & 20.31(19.15, 21.47) & 20.98) & 19.62(18.52, 20.62) & $\begin{array}{l}\text { 19.1(17.98, 20.11) } \\
\text { 23.89(22.05, }\end{array}$ \\
\hline $99.9 \%$ & 24.95(23.04,26.81) & 24.6(22.78, 26.31) & $24.33(22.65,26.03)$ & 25.43) \\
\hline -Log likelihood & $\begin{array}{l}2843.00 \\
(2796.63,2889.72)\end{array}$ & $\begin{array}{l}2843.21 \\
(2799.86,2891.41)\end{array}$ & $\begin{array}{l}2843.57 \\
(2795.53,2887.36) \\
\end{array}$ & $\begin{array}{l}2844.96 \\
(2796.74 \\
2890.19)\end{array}$ \\
\hline
\end{tabular}

